\title{
QCD evolution equations at small $x$ : Pomerons, Odderons and more
}

\author{
Jamal Jalilian-Marian ${ }^{1,2, a}$ \\ ${ }^{1}$ Natural Sciences Department, Baruch College, 17 Lexington Ave., New York, NY 10010, USA \\ ${ }^{2}$ CUNY Graduate Center, 365 Fifth Ave., New York, NY 10016, USA
}

\begin{abstract}
We give an overview of QCD at small $x$, and the equation that governs evolution of observables with energy (or $x$ ), knows as the JIMWLK equation. We show that it reduces, in the appropriate limits, to all the known perturbative evolution equations at small $x$.
\end{abstract}

\section{Introduction}

Quantum ChromoDynamics (QCD) is an extremely rich and at the same time complicated theory of strong interactions between quarks and gluons, the colored fundamental constituents of matter. Due to its complexity, often one is forced to resort to approximations in order to perform theoretical calculations of physical observables. One such approximation is perturbation theory where one expands the expressions for the observables in a power series in the coupling constant. Due to the non-Abelian nature of QCD and asymptotic freedom, the running coupling constant $\alpha_{s}$ becomes small at high momentum transfer $p_{t}$. Perturbative QCD (pQCD) is a systematic approach to particle production in QCD at high $p_{t}$. The justification for applying pQCD to scattering and production of strongly interacting particles lies on the proof and use of collinear factorization theorems which guarantee a clean separation of short and long distance physics effects. In the collinear factorization formalism, particle production cross sections are written as a convolution of parton distribution functions $x f$, nonperturbative yet universal quantities describing the momentum distribution of partons in a nucleon, of the incoming hadrons/nuclei with the hard scattering matrix elements $d \sigma^{\text {parton }}$ computable in pQCD to any order in the strong coupling $\alpha_{s}$. This can symbolically written as

$$
d \sigma^{\text {hadron }} \sim x f_{1} \otimes x f_{2} \otimes d \sigma^{\text {parton }} \otimes z D
$$

where fragmentation function $z D$ describes hadronization; how a colored parton (quark or gluon) turns into a colorless hadron. The fact that these parton distributions are universal (process independent) means they can be measured in one process, say Deep Inelastic Scattering (DIS) of electrons on a nucleon, and used in the above formula to predict the cross section for particle production in other processes. This universality of parton distribution functions is responsible for the predictive power of pQCD. Experimentally observed spectrum of produced particle in various experiments are in excellent

\footnotetext{
ae-mail: jamal.jalilian-marian@baruch.cuny.edu
} 
agreement with predictions of QCD based on collinear factorization over many orders of transverse momenta.

The parton distribution functions $x f$ which are an essential ingredient in a collinear factorization theorem depend on and evolve with scale $Q^{2}$ as described by the DGLAP evolutions equations (see [1] for a review). This "evolution" is due to radiation of extra partons as allowed by the available kinematic phase space in $Q^{2}$. As quarks can radiate gluons and gluons radiate other gluons (and quark anti-quark pairs), the DGLAP equation is a coupled differential-integral equation which can symbolically written as

$$
\frac{d}{d \log Q^{2}}\left(\begin{array}{c}
x q \\
x G
\end{array}\right)=\left(\begin{array}{cc}
P_{q q} & P_{q g} \\
P_{g q} & P_{g g}
\end{array}\right) \otimes\left(\begin{array}{c}
x q \\
x G
\end{array}\right)
$$

where $\otimes$ stands for integration over momentum fraction $x$ and $P_{q q}, \cdots$ are the so-called splitting functions describing the probability for a parton to radiate another one and are calculated in pQCD to any order in the coupling constant. As this is a differential equation one needs initial conditions for the quark and gluon distribution functions $x q, x g$ at some initial scale $Q_{0}^{2}$ (and at all $x$ ), typically taken to be of order of $1 \mathrm{GeV}^{2}$, after which one can solve the DGLAP equation (2) to obtain the distribution functions $x q\left(x, Q^{2}\right), x g\left(x, Q^{2}\right)$ which can then be used in the production cross section as described in the collinear factorization approach.

DGLAP evolution equation predicts a sharp rise in the (sea) quark and gluon distribution functions as the $Q^{2}$ or $1 / x$ increases. There are more and more partons in the wave unction of a hadron as $Q^{2}$ increases, however each parton has an effective size of $1 / Q^{2}$ so that partons which are radiated are occupying smaller and smaller areas. The number of prtons also increases when one increases $1 / x$ as can be seen by noting that $P_{g g} \rightarrow \frac{1}{x}$ as $x \rightarrow 0$. However here there is a profound difference; at fixed $Q^{2}$, as one decreases $x$ (the fraction of the hadron energy carried by a parton) more and more partons are radiated into the same effective area and will start to "overlap". This would lead to an interaction among these partons which are treated as quasi-free in the DGLAP formalism and lead to its breakdown. This untamed growth of parton distribution function would also lead to an unbounded growth of perturbative ross section and an eventual violation of Froissart (unitary) bound. Therefore one expects new QCD dynamics to become manifest when probing a hadronic/nuclear wave function small $x$.

This idea that gluons at small $x$ will start to overlap and that the DGLAP equation would need to be modified was first pointed out by Gribov, Levin and Ryskin (GLR) (see [2] for reviews and comprehensive references) who pointed out that there should be a saturation of gluon density at small enough $x$ such that the growth of perturbative cross sections are tamed. Based on this idea Mueller and Qiu calculated the Feynman diagrams leading to gluon saturation at small $\mathrm{x}$ and derived a new evolution equation for gluon distribution function which can be written symbolically as

$$
\frac{d}{d \log Q^{2}} x G\left(x, Q^{2}\right) \sim x G\left(x, Q^{2}\right)-x G^{2}\left(x, Q^{2}\right)
$$

The second term is due to "recombination" of gluons and comes with a negative sign; it will lead to a slowdown and eventual saturation of the gluon distribution function. Later McLerran and Venugopalan $[3,4]$ proposed an effective action approach to gluon saturation, they introduced a new scale, the saturation momentum $Q_{s}$ and argued that this scale can be much larger than the genuinely nonperturbative scale in $\mathrm{QCD}, \Lambda_{Q C D}$ at small $x$ and/or for large nuclei. Therefore, they reasoned that one could apply weak coupling techniques to address gluon saturation even though one deals with a manybody system of interacting gluons. They realized that the relevant time scales are very different for small and large $x$ degrees of freedom. Utilizing this they introduced the notion of color charge density 
$\rho^{a}$ to represent the color charges at large $x$ which in turn generate a classical color field describing the small $x$ degrees of freedom. As one is interested in the small $x$ limit, the coupling between the color charges $\rho^{a}$ and the classical field is taken to be eikonal.

The McLerran-Venugopalan (MV) effective action can be written as

$$
\begin{aligned}
S \equiv & -\frac{1}{4} \int d^{4} x F_{\mu \nu}^{a 2}(x)+\frac{i}{N_{c}} \int d^{2} x d x^{-} \operatorname{Tr} \rho\left(x^{-}, x_{t}\right) W_{-\infty, \infty}\left[A^{-}\right]\left(x^{-}, x_{t}\right) \\
& +i \int d^{2} x d x^{-} F\left[\rho\left(x_{t}, x^{-}\right)\right]
\end{aligned}
$$

where

$$
W_{-\infty, \infty}\left[A^{-}\right]\left(x^{-}, x_{t}\right) \equiv \operatorname{Pexp}\left[-i g \int_{-\infty}^{\infty} d x^{+} A_{a}^{-}\left(x^{+}, x^{-}, x_{t}\right) T_{a}\right]
$$

and

$$
\exp \left[i\left\{i \int d^{2} x d x^{-} F\left[\rho\left(x_{t}, x^{-}\right)\right]\right\}\right]
$$

can be thought as the statistical weight of a given color charge configuration. In the original MV model, $F$ was taken to be a Gaussian, given by

$$
\int d x^{-} F\left[\rho\left(x_{t}, x^{-}\right)\right]=\frac{1}{2 \mu^{2}} \rho^{2}\left(x_{t}\right)
$$

In the MV effective action approach one first solves the classical equation of motion for a given configuration of color current $J_{a}^{\mu}(x)=\delta^{\mu+} \delta\left(x^{-}\right) \rho_{a}$

$$
D_{\mu} F_{a}^{\mu v}=J_{a}^{v}
$$

Given $A^{\mu}[\rho]$, the solution of the classical equation of motion (8) for a given color charge configuration one then averages over all color charges using the statistical weight given in (6) in order to compute a physical observable which typically involves correlations of the gluon field (or Wilson lines involving the gluon fields).

The procedure described above is valid at the classical level and needs to be augmented with quantum loop effects. In the kinematics of interest (high energy) this corresponds to a resummation of loop diagrams which are divergent in the limit $x \rightarrow 0$. This can be physically understood as including more of the small $x$ degrees of freedom into our definition of color charges at large $x$ which leads to a renormalization of the color charge $\rho$, or more accurately, a renormalization of the weight functional describing the distribution of color charges $\rho$. This resummation of small $x$ effects can be done at the effective action level, and consequently for the $x$ evolution of any correlation function computed using this effective action. The resulting equation is known as the JIMWLK evolution equation [5] which is written as

$$
\frac{\partial}{d \log 1 / x}<O>_{x}=<H O>_{x}
$$

where the Hamiltonian $H$ is

$$
H=-\frac{1}{2(2 \pi)^{3}} \int d^{2} x_{t} d^{2} y_{t} d^{2} z_{t} M_{x y z}\left(1+U_{x}^{\dagger} U_{y}-U_{x}^{\dagger} U_{z}-U_{z}^{\dagger} U_{y}\right)^{a b} \frac{\delta}{\delta \alpha_{x}^{a}} \frac{\delta}{\delta \alpha_{y}^{b}},
$$


Here $x_{t}, y_{t}, z_{t}$ are two-dimensional vectors on the transverse plane and $a$ and $b$ are color indices. The dipole kernel $M_{x y z}$ is defined as

$$
M_{x y z} \equiv \frac{\left(x_{t}-y_{t}\right)^{2}}{\left(x_{t}-z_{t}\right)^{2}\left(z_{t}-y_{t}\right)^{2}}
$$

The Wilson line $U_{z}$ is in the adjoint representation and is given by

$$
U_{z} \equiv e^{-i g \alpha^{a}\left(z_{t}\right) t^{a}},
$$

where $\alpha^{a}\left(z_{t}\right)$ is the classical solution. Given the JIMWLK equation (9) one can derive evolution equations for any correlation function in the theory. The most ubiquitous one is the so called dipole cross section, defined as the normalized trace of Wilson lines (in the fundamental representation). It is

$$
\frac{d}{d y}\left\langle\operatorname{tr} V_{r}^{\dagger} V_{\bar{r}}\right\rangle=-\frac{N_{c} \alpha_{s}}{2 \pi^{2}} \int d^{2} z \frac{(r-\bar{r})^{2}}{(r-z)^{2}(\bar{r}-z)^{2}}\left\langle\operatorname{tr} V_{r}^{\dagger} V_{\bar{r}}-\frac{1}{N_{c}} \operatorname{tr} V_{r}^{\dagger} V_{z} \operatorname{tr} V_{\bar{r}} V_{z}^{\dagger}\right\rangle .
$$

with $y \equiv \log 1 / x$. This equation reduced to the BK equation [6] after making a Gaussian approximation after which it can be written as

$$
\frac{d}{d y} S(r-s)=-\frac{N_{c} \alpha_{s}}{2 \pi^{2}} \int d^{2} z \frac{(r-s)^{2}}{(r-z)^{2}(s-z)^{2}}[S(r-s)-S(r-z) S(z-s)]
$$

where the $S$ matrix is defined as

$$
\langle S(r-\bar{r})\rangle \equiv \frac{1}{N_{c}}\left\langle\operatorname{tr} V_{r} V_{\bar{r}}^{\dagger}\right\rangle
$$

BK equation is the simplest non-linear equation which contains the dynamics of gluon saturation. Expanded in powers of the gluon field $g A^{\mu}$ and keeping the first non-trivial term it reduces to the famous BFKL equation [7] for the evolution of gluonic two-point function. The BFKL equation sums the ladder diagrams where the rapidity $(x)$ variable is ordered along the ladder. This is the so-called (perturbative or BFKL) pomeron which corresponds to the state of two Reggeized gluons exchanged in the $t$ channel. Using the JIMWLK equation (9) one can also derive a evolution equation for the state of 3 or more Reggeized gluons where state of 3 Reggeized gluons in $t$ channel is given its own name and is usually referred to as a odderon. For exchange of 4 or more Reggeized gluons one can show that defining the $T$ matrix for the $2 n$-point function

$$
T^{(2 n)}\left(x_{1}, x_{2}, \cdots x_{2 n}\right)=1-\frac{1}{N_{c}} \operatorname{Tr}\left(V_{x_{1}} V_{x_{2}}^{\dagger} V_{x_{3}} V_{x_{4}}^{\dagger} \cdots V_{x_{2 n-1}} V_{x_{2 n}}^{\dagger}\right)
$$

one gets (in the linear region where all Wilson lines are expanded) [8]

$$
\begin{aligned}
& \frac{d}{d y} T^{(2 n)}\left(l_{1}, l_{2}, \cdots l_{2 n-1}, l_{2 n}\right)=-\frac{\bar{\alpha}}{2 \pi} \sum_{j=1}^{2 n} \int d^{2} p_{t}\left[\frac{l_{j}^{2}}{p_{t}^{2}\left[p_{t}^{2}+\left(p_{t}-l_{j}\right)^{2}\right]}\right] T_{\left(\prod_{k=1}^{2 n} l_{k}\right)}^{(2 n)} \\
+ & \frac{\bar{\alpha}}{4 \pi} \int d^{2} p_{t}\left[\frac{l_{1}^{2}}{p_{t}^{2}\left(p_{t}+l_{1}\right)^{2}}+\frac{l_{2 n}^{2}}{p_{t}^{2}\left(p_{t}-l_{2 n}\right)^{2}}-\frac{\left(l_{1}+l_{2 n}\right)^{2}}{\left(p_{t}+l_{1}\right)^{2}\left(p_{t}-l_{2 n}\right)^{2}}\right] T_{\left(l_{1}+p_{t}\right)\left(\prod_{k=2}^{2 n-1} l_{k}\right)\left(l_{2 n}-p_{t}\right)}^{(2 n)} \\
+ & \frac{\bar{\alpha}}{4 \pi} \sum_{j=2}^{2 n} \int d^{2} p_{t}\left[\frac{l_{j-1}^{2}}{p_{t}^{2}\left(p_{t}+l_{j-1}\right)^{2}}+\frac{l_{j}^{2}}{p_{t}^{2}\left(p_{t}-l_{j}\right)^{2}}-\frac{\left(l_{j-1}+l_{j}\right)^{2}}{\left(p_{t}+l_{j-1}\right)^{2}\left(p_{t}-l_{j}\right)^{2}}\right] T_{\left(\prod_{k=1}^{j-2} l_{k}\right)\left(l_{j-1}+p_{t}\right)\left(l_{j}-p_{t}\right)\left(\prod_{k=j+1}^{2 n} l_{k}\right)}^{(2 n)}
\end{aligned}
$$


Eq. (17) describes evolution of a state of $2 n$ Reggeized gluons exchanged in the $t$ channel and is identical to the BJKP equation [9]. We emphasize that the JIMWLK equation for the $2 n$-point function in general includes non-linear terms; here we have ignored the non-linearities of the JIMWLK equation to demonstrate that it includes the BJKP equation as its linear limit (in the region where the gluon field strength is not large). The full non-linear evolution equation for $2 n$-point function can be written as [8]

$$
H \hat{T}^{(2 n)}=\frac{\bar{\alpha}}{4 \pi} \int d^{2} z_{t}\left[H_{1} \hat{T}^{(2 n)}+H_{2} \hat{T}^{(2 n)}-H_{3} \hat{T}^{(2 n)}-H_{4} \hat{T}^{(2 n)}\right]
$$

where $H_{1,2,3,4}$ act on $\hat{T}^{(2 n)}$ as

$$
\begin{aligned}
& H_{1} \hat{S}^{(2 n)}=-\sum_{i=1}^{n}\left[M_{x_{2 i-1} x_{2 i} z}\right] \hat{S}_{\left(\prod_{k=1}^{2 n} x_{k}\right)}^{(2 n)} \\
+ & \sum_{j=2 ; j>i}^{n} \sum_{i=1}^{n-1}\left[M_{x_{2 i-1} x_{2 j-1} z}-M_{x_{2 i-1} x_{2 j} z}-M_{x_{2 i} x_{2 j-1} z}+M_{x_{2 i} x_{2 j} z}\right] \hat{S}_{\left(\prod_{k=2 i}^{2 j-1} x_{k}\right)}^{(2 j-2 i)} \hat{S}_{\left(\prod_{k=1}^{2 i-1} x_{k}\right)\left(\prod_{k=2 j}^{2 n} x_{k}\right)}^{(2 n-2 j+2 i)}
\end{aligned}
$$

and

$$
\begin{aligned}
H_{2} \hat{S}^{(2 n)}= & -\sum_{i=1}^{n}\left[M_{x_{2 i-1} x_{2 i} z}\right] \hat{S}_{\left(x_{2 i-1} x_{2 i}\right)}^{(2)} \hat{S}_{\left(\prod_{k=1}^{2 i-2} x_{k}\right)\left(\prod_{k=2 i+1}^{2 n} x_{k}\right)}^{(2 n-2)} \\
& +\sum_{j=2 ; j>i}^{n} \sum_{i=1}^{n-1}\left[M_{x_{2 i-1} x_{2 j-1} z}\right] \hat{S}_{\left(\prod_{k=2 i-1}^{2 j-2} x_{k}\right)}^{(2 j-2 i)} \hat{S}_{\left(\prod_{k=1}^{2 i-2} x_{k}\right)\left(\prod_{k=2 j-1}^{2 n} x_{k}\right)}^{(2 n-2 j+2 i)} \\
& -\sum_{j=2 ; j>i}^{n} \sum_{i=1}^{n-1}\left[M_{x_{2 i-1} x_{2 j} z}\right] \hat{S}_{\left(\prod_{k=2 i-1}^{2 j} x_{k}\right)}^{(2 j-2 i+2)} \hat{S}_{\left(\prod_{k=1}^{2 i-2} x_{k}\right)\left(\prod_{k=2 j+1}^{2 n} x_{k}\right)}^{(2 n-2 j+2 i-2)} \\
& -\sum_{j=2 ; j>i}^{n} \sum_{i=1}^{n-1}\left[M_{x_{2 i} x_{2 j-1} z}\right] \hat{S}_{\left(\prod_{k=2 i+1}^{2 j-2} x_{k}\right)}^{(2 j-2 i-2)} \hat{S}_{\left(\prod_{k=1}^{2 i} x_{k}\right)\left(\prod_{k=2 j-1}^{2 n} x_{k}\right)}^{(2 n-2 j+2 i+2)} \\
& +\sum_{j=2 ; j>i}^{n} \sum_{i=1}^{n-1}\left[M_{x_{2 i} x_{2 j} z}\right] \hat{S}_{\left(\prod_{k=2 i+1}^{2 j} x_{k}\right)}^{(2 j-2 i)} \hat{S}_{\left(\prod_{k=1}^{2 i} x_{k}\right)\left(\prod_{k=2 j+1}^{2 n} x_{k}\right)}^{(2 n-2 j+2 i)}
\end{aligned}
$$


and

$$
\begin{aligned}
H_{3} \hat{S}^{(2 n)}= & -\sum_{i=1}^{n}\left[M_{x_{2 i-1} x_{2 i} z}\right] \frac{1}{2} \hat{S}_{z x_{2 i-1}}^{(2)} \hat{S}_{\left(\prod_{k=1}^{2 i-2} x_{k}\right) z\left(\prod_{k=2 i}^{2 n} x_{k}\right)}^{(2 n)} \\
& -\sum_{i=1}^{n}\left[M_{x_{2 i-1} x_{2 i} z}\right] \frac{1}{2} \hat{S}_{z x_{2 i}}^{(2)} \hat{S}_{\left(\prod_{k=1}^{2 i-1} x_{k}\right) z\left(\prod_{k=2 i+1}^{2 n} x_{k}\right)}^{(2 n)} \\
& +\sum_{j=2 ; j>i}^{n} \sum_{i=1}^{n-1}\left[M_{x_{2 i-1} x_{2 j-1} z}-M_{x_{2 i-1} x_{2 j} z}\right] \frac{1}{2} \hat{S}_{z\left(\prod_{k=2 i-1}^{2 j-1} x_{k}\right)}^{(2 j-2 i+2)} \hat{S}_{\left(\prod_{k=1}^{2 i-2} x_{k}\right) z\left(\prod_{k=2 j}^{2 n} x_{k}\right)}^{(2 n-2 j+2 i)} \\
& +\sum_{j=2 ; j>i}^{n} \sum_{i=1}^{n-1}\left[-M_{x_{2 i} x_{2 j-1} z}+M_{x_{2 i} x_{2 j} z}\right] \frac{1}{2} \hat{S}_{z\left(\prod_{k=2 i+1}^{2 j-1} x_{k}\right)}^{(2 j-2 i)} \hat{S}_{\left(\prod_{k=1}^{2 i} x_{k}\right) z\left(\prod_{k=2 j}^{2 n} x_{k}\right)}^{(2 n-2 j+2 i+2)} \\
& +\sum_{j=2 ; j>i}^{n} \sum_{i=1}^{n-1}\left[M_{x_{2 j-1} x_{2 i-1} z}-M_{x_{2 j-1} x_{2 i} z}\right] \frac{1}{2} \hat{S}_{z\left(\prod_{k=2 i}^{2 j-2} x_{k}\right)}^{(2 j-2 i)} \hat{S}_{\left(\prod_{k=1}^{2 n-1} x_{k}\right) z\left(\prod_{k=2 j-1}^{2 n} x_{k}\right)}^{(2 n-2 j+2 i+2)} \\
& +\sum_{j=2 ; j>i}^{n} \sum_{i=1}^{n-1}\left[-M_{x_{2 j} x_{2 i-1} z}+M_{x_{2 j} x_{2 i} z}\right] \frac{1}{2} \hat{S}_{z\left(\prod_{k=2 i}^{2 j} x_{k}\right)}^{(2 j-2 i+2)} \hat{S}_{\left(\prod_{k=1}^{2 n-1} x_{k}\right) z\left(\prod_{k=2 j+1}^{2 n} x_{k}\right)}^{(2 n-2 j+2 i)}
\end{aligned}
$$

and

$$
\begin{aligned}
H_{4} \hat{S}^{(2 n)}= & -\sum_{i=1}^{n}\left[M_{x_{2 i-1} x_{2 i} z}\right] \frac{1}{2} \hat{S}_{z x_{2 i}}^{(2)} \hat{S}_{\left(\prod_{k=1}^{2 i-1} x_{k}\right) z\left(\prod_{k=2 i+1}^{2 n} x_{k}\right)}^{(2 n)} \\
& -\sum_{i=1}^{n}\left[M_{x_{2 i-1} x_{2 i} z}\right] \frac{1}{2} \hat{S}_{z x_{2 i-1}}^{(2)} \hat{S}_{\left(\prod_{k=1}^{2 i-2} x_{k}\right) z\left(\prod_{k=2 i}^{2 n} x_{k}\right)}^{(2 n)} \\
& +\sum_{j=2 ; j>i}^{n} \sum_{i=1}^{n-1}\left[M_{x_{2 i-1} x_{2 j-1} z}-M_{x_{2 i} x_{2 j-1} z}\right] \frac{1}{2} \hat{S}_{z\left(\prod_{k=2 i}^{2 j-2} x_{k}\right)}^{(2 j-2 i)} \hat{S}_{\left(\prod_{k=1}^{2 n-1} x_{k}\right) z\left(\prod_{k=2 j-1}^{2 n} x_{k}\right)}^{(2 n-2 j+2 i+2)} \\
& +\sum_{j=2 ; j>i}^{n} \sum_{i=1}^{n-1}\left[-M_{x_{2 i-1} x_{2 j} z}+M_{x_{2 i} x_{2 j} z}\right] \frac{1}{2} \hat{S}_{z\left(\prod_{k=2 i}^{2 j} x_{k}\right)}^{(2 j-2 i+2)} \hat{S}_{\left(\prod_{k=1}^{2 n-1} x_{k}\right) z\left(\prod_{k=2 j+1}^{2 n} x_{k}\right)}^{(2 n-2 j+2 i)} \\
& +\sum_{j=2 ; j>i}^{n} \sum_{i=1}^{n-1}\left[M_{x_{2 j-1} x_{2 i-1} z}-M_{x_{2 j} x_{2 i-1} z}\right] \frac{1}{2} \hat{S}_{z\left(\prod_{k=2 i-1}^{2 j-1} x_{k}\right)}^{(2 j-2 i+2)} \hat{S}_{\left(\prod_{k=1}^{2 n-2} x_{k}\right) z\left(\prod_{k=2 j}^{2 n} x_{k}\right)}^{(2 n-2 j+2 i)} \\
& +\sum_{j=2 ; j>i}^{n} \sum_{i=1}^{n-1}\left[-M_{x_{2 j-1} x_{2 i} z}+M_{x_{2 j} x_{2 i} z}\right] \frac{1}{2} \hat{S}_{z\left(\prod_{k=2 i+1}^{2 j-1} x_{k}\right)}^{(2 j-2 i)} \hat{S}_{\left(\prod_{k=1}^{2 i} x_{k}\right) z\left(\prod_{k=2 j}^{2 n} x_{k}\right)}^{(2 n-2 j+2 i+2)}
\end{aligned}
$$

It is not possible, in principle, to solve these equations analytically. Therefore either one has to make further approximations or resort to numerical techniques. The most common approximation being the Gaussian truncation which allows one to write all higher point functions in terms of the two point function $S$ which satisfies the BK equation,

$$
S^{(2 n)} \simeq S^{n}
$$

where $S^{(2 n)}=1-T^{(2 n)}$ and $T^{(2 n)}$ is defined in (16). The dipole cross section $S\left(r_{t}=x_{t}-y_{t}\right)$ goes to zero as $r_{t} \rightarrow 0$ (color transparency) and approaches 1 as $r_{t} \rightarrow \infty$ (black disk limit). The dipole cross section is the ubiquitous quantity appearing in the DIS structure functions $F_{2}, F_{L}$ and in single 
inclusive particle production in the forward rapidity region of hadron-hadron (nucleus) collisions [10]. Higher point functions such as the Quadrupole [11], normalized trace of 4 Wilson lines, on the other hand appear in less inclusive quantities such as di-hadron production in DIS and in proton-nucleus collisions. The evolution of Quadrupole has been studied numerically and has been shown to grow with the same power of energy $(x)$ and exhibit geometric scaling as the dipole cross section does. The small $x$ evolution equations are not closed form equations; evolution of a $2 n$-point function involves higher point functions [12]; however it can be shown that with leading $N_{c}$ accuracy they involve only dipoles and quadrupoles. All other higher point functions are suppressed bu powers of $N_{c}$.

In summary, the effective action approach to QCD at small $x$ has enabled one to study the dynamics of QCD at small $x$ systematically and in a theoretically controlled way. Using this effective action a non-linear evolution equation known as the JIMWLK equation has been derived which describes the $x$ (or equivalently energy or rapidity) dependence of $2 n$-point functions of Wilson lines which are the ingredients of particle production cross sections in high energy hadronic collisions. It has been shown that the JIMWLK equation for $2 n$-point function of Wilson lines reduces to the BFKL and BJKP equations in the linear (dilute) regime. While there has been recent progress in computing higher order corrections to the JIMWLK equation, yet the ultimate goal, to include the physics of DGLAP evolution, remains to be realized.

\section{References}

[1] H. L. Lai, J. Botts, J. Huston, J. G. Morfin, J. F. Owens, J. w. Qiu, W. K. Tung and H. Weerts, Phys. Rev. D 51, 4763 (1995) doi:10.1103/PhysRevD.51.4763.

[2] H. Weigert, Prog. Part. Nucl. Phys. 55, 461 (2005); F. Gelis, E. Iancu, J. Jalilian-Marian, R. Venugopalan, Ann. Rev. Nucl. Part. Sci. 60, $463-489$ (2010). [arXiv:1002.0333 [hep-ph]]; J. JalilianMarian, Y. V. Kovchegov, Prog. Part. Nucl. Phys. 56, 104-231 (2006); J. L. Albacete and C. Marquet, Prog. Part. Nucl. Phys. 76, 1 (2014).

[3] L. D. McLerran and R. Venugopalan, Phys. Rev. D 49, 2233 (1994), Phys. Rev. D 49, 3352 (1994); J. Jalilian-Marian, A. Kovner, L. D. McLerran and H. Weigert, Phys. Rev. D 55, 5414 (1997).

[4] S. Jeon and R. Venugopalan, Phys. Rev. D 71, 125003 (2005); A. Dumitru, J. Jalilian-Marian, E. Petreska, Phys. Rev. D84, 014018 (2011); J. Jalilian-Marian, S. Jeon and R. Venugopalan, Phys. Rev. D 63, 036004 (2001); A. Ayala, J. Jalilian-Marian, L. D. McLerran and R. Venugopalan, Phys. Rev. D 52, 2935 (1995) doi:10.1103/PhysRevD.52.2935.

[5] J. Jalilian-Marian, A. Kovner, L. D. McLerran and H. Weigert, Phys. Rev. D 55, 5414 (1997); J. Jalilian-Marian, A. Kovner, A. Leonidov and H. Weigert, Nucl. Phys. B 504, 415 (1997), Phys. Rev. D 59, 014014 (1999), Phys. Rev. D 59, 014015 (1999), Phys. Rev. D 59, 034007 (1999), A. Kovner, J. G. Milhano and H. Weigert, Phys. Rev. D 62, 114005 (2000); A. Kovner and J. G. Milhano, Phys. Rev. D 61, 014012 (2000); E. Iancu, A. Leonidov and L. D. McLerran, Nucl. Phys. A 692, 583 (2001), Phys. Lett. B 510, 133 (2001); E. Ferreiro, E. Iancu, A. Leonidov and L. McLerran, Nucl. Phys. A 703, 489 (2002).

[6] I. Balitsky, Nucl. Phys. B 463, 99 (1996); Y. V. Kovchegov, Phys. Rev. D 60, 034008 (1999); Phys. Rev. D 61, 074018 (2000).

[7] L. N. Lipatov, Sov. J. Nucl. Phys. 23, 338 (1976) [Yad. Fiz. 23, 642 (1976)]; E. A. Kuraev, L. N. Lipatov and V. S. Fadin, Sov. Phys. JETP 45, 199 (1977) [Zh. Eksp. Teor. Fiz. 72, 377 (1977)]; I. I. Balitsky and L. N. Lipatov, Sov. J. Nucl. Phys. 28, 822 (1978) [Yad. Fiz. 28, 1597 (1978)]. 
[8] A. Ayala, E. R. Cazaroto, L. A. Hernández, J. Jalilian-Marian and M. E. Tejeda-Yeomans, Phys. Rev. D 90, no. 7, 074037 (2014) doi:10.1103/PhysRevD.90.074037; J. Jalilian-Marian, Phys. Rev. D 85, 014037 (2012) doi:10.1103/PhysRevD.85.014037.

[9] J. Bartels, Nucl. Phys. B 175, 365 (1980); T. Jaroszewicz, Acta Phys. Polon. B 11, 965 (1980); J. Kwiecinski and M. Praszalowicz, Phys. Lett. B 94, 413 (1980); Z. Chen, A. H. Mueller, Nucl. Phys. B451, 579-604 (1995).

[10] A. Dumitru and J. Jalilian-Marian, Phys. Rev. Lett. 89, 022301 (2002); D. Kharzeev, Y. V. Kovchegov and K. Tuchin, Phys. Rev. D 68, 094013 (2003); J. L. Albacete, N. Armesto, A. Kovner, C. A. Salgado and U. A. Wiedemann, Phys. Rev. Lett. 92, 082001 (2004); J. Jalilian-Marian, Y. Nara and R. Venugopalan, Phys. Lett. B 577, 54 (2003) doi:10.1016/j.physletb.2003.09.097; J. Jalilian-Marian, J. Phys. G 30, S751 (2004), Nucl. Phys. A 748, 664 (2005), J. Jalilian-Marian, Nucl. Phys. A 753, 307 (2005) doi:10.1016/j.nuclphysa.2005.02.156, Phys. Rev. C 70, 027902 (2004), Nucl. Phys. A 739, 319 (2004); A. Dumitru, A. Hayashigaki and J. Jalilian-Marian, Nucl. Phys. A 765, 464 (2006), Nucl. Phys. A 770, 57 (2006); E. M. Henley and J. Jalilian-Marian, Phys. Rev. D 73, 094004 (2006) doi:10.1103/PhysRevD.73.094004;

F. Gelis and J. Jalilian-Marian, Phys. Rev. D 76, 074015 (2007), Phys. Rev. D 67, 074019 (2003) doi:10.1103/PhysRevD.67.074019, Phys. Rev. D 66, 094014 (2002) doi:10.1103/PhysRevD.66.094014, Phys. Rev. D 66, 014021 (2002) doi:10.1103/PhysRevD.66.014021;

D. Boer, A. Utermann and E. Wessels, Phys. Rev. D 77, 054014 (2008); E. R. Cazaroto, V. P. Gonçalves, F. S. Navarra, Nucl. Phys. A 872, 196 (2011); J. Jalilian-Marian and A. H. Rezaeian, Phys. Rev. D 85, 014017 (2012), J. Jalilian-Marian and A. H. Rezaeian, Phys. Rev. D 86, 034016 (2012); A. H. Rezaeian, Phys. Rev. D 86, 094016 (2012).

[11] J. Jalilian-Marian and Y. V. Kovchegov, Phys. Rev. D 70, 114017 (2004) [Erratum-ibid. D 71, 079901 (2005)]; A. Kovner and M. Lublinsky, JHEP 0611, 083 (2006), Phys. Rev. D 84, 094011 (2011), Phys. Rev. D 83, 034017 (2011); J. Jalilian-Marian, Nucl. Phys. A 770, 210 (2006); C. Marquet, Nucl. Phys. A 796, 41 (2007); J. L. Albacete and C. Marquet, Phys. Rev. Lett. 105, 162301 (2010).

[12] Y. V. Kovchegov, J. Kuokkanen, K. Rummukainen and H. Weigert, Nucl. Phys. A 823, 47 (2009); C. Marquet and H. Weigert, Nucl. Phys. A 843, 68 (2010); A. Dumitru and J. JalilianMarian, Phys. Rev. D 82, 074023 (2010), Phys. Rev. D 81, 094015 (2010); A. Dumitru, J. JalilianMarian, T. Lappi, B. Schenke and R. Venugopalan, Phys. Lett. B 706, 219 (2011); A. Dumitru, K. Dusling, F. Gelis, J. Jalilian-Marian, T. Lappi and R. Venugopalan, Phys. Lett. B 697, 21 (2011) doi:10.1016/j.physletb.2011.01.024; A. Ayala, M. Hentschinski, J. Jalilian-Marian and M. E. Tejeda-Yeomans, Phys. Lett. B 761, 229 (2016) doi:10.1016/j.physletb.2016.08.035. 